\title{
Growth rates of ITG modes in the presence of flow shear
}

\author{
V.I. Dagnelie, ${ }^{1,2}$ J. Citrin, ${ }^{1}$ F. Jenko, ${ }^{3}$ M.J. Pueschel, $,{ }^{4}, 5$ T. Görler,${ }^{3}$ D. Told,${ }^{3}$ and H. Doerk ${ }^{3}$ \\ ${ }^{1}$ DIFFER - Dutch Institute for Fundamental Energy Research, \\ De Zaale 20, 5612AJ Eindhoven, The Netherlands \\ ${ }^{2}$ Institute for Theoretical Physics, Utrecht University, \\ Princetonplein 5, 3584CC Utrecht, The Netherlands \\ ${ }^{3}$ Max Planck Institute for Plasma Physics, Boltzmannstr. 2, 85748 Garching, Germany \\ ${ }^{4}$ Institute for Fusion Studies, University of Texas at Austin, Austin, Texas 78712, USA \\ ${ }^{5}$ Department of Physics, University of Wisconsin-Madison, Madison, Wisconsin 53706, USA
}

\begin{abstract}
Plasma microinstabilities in toroidal magnetic confinement devices can be driven unstable by a radial ion temperature gradient, and stabilized by rotational flow shear. In this study we argue that these nonlinear dynamics can be captured by the linear stabilization of Floquet modes. To that end, we propose a novel method (the $\tau_{\mathrm{AC}}$ method) to calculate growth rates by averaging over linear Floquet modes. The $\tau_{\mathrm{AC}}$ method is compared to nonlinear and other linear approaches, and is shown to work well at low parallel velocity gradient drive. As such, the method provides a promising approach to explore the parameter dependencies of flow shear stabilization.
\end{abstract}

\section{INTRODUCTION}

Turbulent transport is the primary mechanism which limits plasma core confinement in tokamaks [1]. Among the main instability branches which drive turbulence are ion-temperature-gradient (ITG) modes [2, 3]. At higher amplitudes, the modes couple nonlinearly and eventually saturate in a statistically quasi-stationary state. However, at transport-driving spatial scales, the linear characteristics of these modes are still evident in the nonlinear state, see Refs. [4-7] and references therein. Correct evaluation of linear mode characteristics is vital for the accuracy of quasilinear transport models.

Confinement improvement through turbulent transport reduction would allow smaller fusion reactors to be built for the same output fusion power. One method to mitigate or weaken the impact of ITG modes is through plasma rotation flow shear. This has been widely studied experimentally, analytically, and computationally [8-15].

In this work, we investigate linear ITG modes through local (flux-tube) gyrokinetic simulations with the GENE code [16]. The reduction of these linear instabilities by rotational flow shear are then explored by employing a novel method to calculate growth rates. Differences with previous approaches are demonstrated, and the new method is shown to yield physically relevant growth rates, thus providing an approach to determine stabilization of ITG modes by flow shear computationally much cheaper than nonlinear simulations.

In Sec. 2, we provide some of the background formalism, and in Sec. 3, the new growth rate calculation method is oulined. Finally, in Sec. 4, linear and nonlinear simulations are done to test the method against other approaches.

\section{THEORETICAL BACKGROUND}

In this study, GENE is used to simulate a circular tokamak on a spectral grid $k_{x}, k_{y}, z$, where $x$ is the radial and $y$ the binormal direction, while the parallel direction is denoted by $z$. GENE normalization [17] is used throughout the work. The linearized gyrokinetic framework [18] is used to calculate the perturbed particle distribution function $f_{1 j}$ of species $j$, with eigenmodes $\Phi\left(k_{\perp}, z, t\right)$.

From $f_{1, j}$ the perturbed plasma density is obtained by $n_{1 j}=\int \mathrm{d}^{3} v f_{1 j}$. The growth rate $\gamma$ of an eigenmode in a time interval $\Delta t$ is then defined as $\frac{n_{1 j}(t+\Delta t)}{n_{1 j}(t)}=e^{\gamma \Delta t}$ (here, the $k_{y}$ dependency is implicit, and an average is taken over $k_{x}$ ). Instead of $n$, other fields and moments can be chosen; all converge to the same growth rate. If this growth rate $\gamma>0$, the corresponding eigenmode $\Phi$ is considered unstable.

To reduce unstable growth rates corresponding to ITG modes, rotational flow shear can be added. In the GENE code, flow shear (defined as $\gamma_{E}=-(x / q) \partial \Omega_{\text {tor }} / \partial x$ with $q$ the safety factor and $\Omega_{\text {tor }}$ the plasma toroidal angular velocity) is implemented by shifting the radial wavenumber grid in time: $k_{x}^{\prime}=k_{x}-k_{y} \gamma_{E} t$, at certain times $t$ $[19,20]$. Because of the finite number of radial modes simulated in a numerical system, the $k_{x}$ shifts are discrete, even though $t$ is a continuous variable. Without flow shear, growth rates converge to a specific value after some time of the simulation. With flow shear, under certain circumstances, so-called Floquet fluctuations occur: the growth rate fluctuates periodically in time, with a fixed amplitude around some average.

\section{THE $\tau_{\mathrm{AC}}$ METHOD}

Stabilization of ITG instabilities by flow shear has been found to adhere well to the so-called quench rule, which states that a mode is stabilized when $\gamma_{E}>\alpha \gamma_{0}$, where $\gamma_{0}$ is the growth rate of the instability at zero flow shear 
and $\alpha$ is a constant [21,22]. As noted by [23], "this is suggestive of an essentially linear process, even though it is a model of the nonlinear simulation results." The linear stabilization of Floquet modes by flow shear has been scrutinized in multiple studies in an attempt to find the underlying linear process of the observed stabilization dynamics. There, effective growth rates from averages over Floquet modes were found to not correspond well to the quench rule as predicted by nonlinear simulations, which has led to the conclusion that the linear approach does not capture the nonlinear stabilization of ITG turbulence $[23,24]$. The effective linear growth rates in the literature, however, are often calculated from a simple average of the fluctuating Floquet mode. Here, we refer to this approach as the $\gamma_{\text {avg }}$ method. Furthermore, studies often use the reduced physics of adiabatic electrons in their simulations. In this work, we argue that by employing kinetic electrons and a novel method to calculate growth rates from Floquet modes, we can capture the nonlinear stabilization of ITG turbulence. (a)

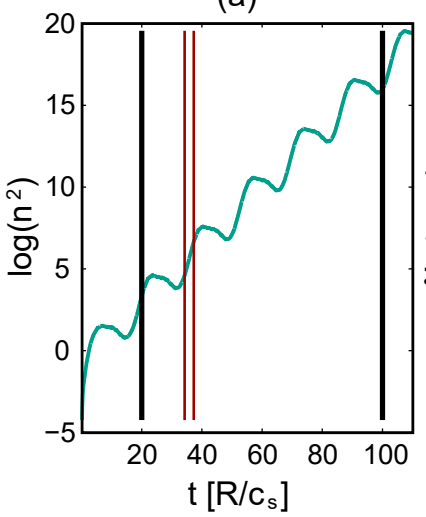

(b)

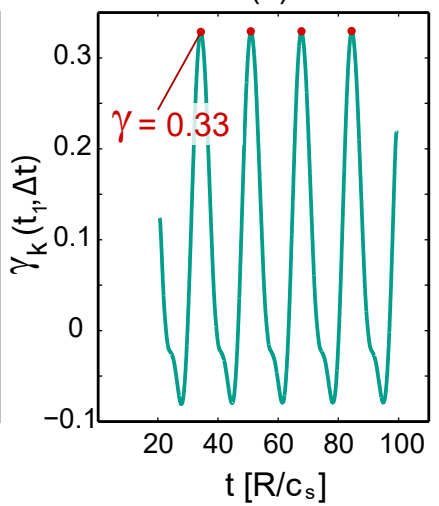

FIG. 1. (a) Mode amplitude timetrace, with growth rates analyzed between the black lines and the calculated converged time window width $\Delta t=1 / \gamma_{k}$ between red lines. (b) Corresponding local growth rates $\gamma_{k}(t 1, \Delta t)$.

To improve upon the $\gamma_{\text {avg }}$ method, we therefore introduce the $\tau_{\mathrm{AC}}$ method, similar to that proposed in Refs. [25, 26]. This approach assumes that the relevant timescale to average over linear growth rates is the nonlinear decorrelation time, thereby inserting a nonlinear element in our otherwise linear approach. Next, it is assumed that this timescale at a given spatial scale $k_{\perp}$ is given by $1 / \gamma_{k}$, where $\gamma_{k}$ is the growth rate of the most unstable mode at the respective spatial scale. Finally, the physical growth rate corresponding to the resulting Floquet-fluctuating timetrace is assumed to be weighted towards the peak of the Floquet cycles, which can intuitively be understood by considering the following. The original instability's energy resides at $k_{x}=0$, where the modes typically have their maximum growth rate. If the nonlinear decorrelation time (itself assumed to be $\left.\sim 1 / \gamma_{k}\right)$ is short enough for a mode not to traverse a full Floquet cycle, it follows that the effective growth rate is still weighted towards the $k_{x}=0$ values.

The proposed method is to iteratively calculate the growth rate using an adaptive time window until the width of the time window converges to the inverse growth rate calculated. This is illustrated in Fig. 1, where a mode amplitude timetrace is shown for a Cyclone Base Case $(\mathrm{CBC})$ linear simulation with kinetic electrons, $\gamma_{E}=0.3$, and $k_{y}=0.3$. CBC parameters are circular geometry, magnetic shear $\hat{s}=\left(r_{0} / q_{0}\right) \partial q / \partial r=0.8$, safety factor $q=1.4$, inverse aspect ratio $\epsilon_{\mathrm{t}}=0.18$, $R / L_{n}=2.2, R / L_{T}=6.75$, where $R$ is the tokamak's major radius, and $L_{n}$ and $L_{T}$ are the scale lengths corresponding to the plasma density and temperature gradients, respectively. In the present work, typical grid sizes are 90 for $k_{x}, 24$ for $z, 32$ for $v_{\|}$and 8 for $\mu$, with $\Delta k_{x} \approx 0.1$. This small $k_{x}$ step size is necessary in the presence of flow shear, when the $k_{x}$ grid itself is coupled to time. At a minimum, $\Delta k_{x}$ must be smaller than the distance between modes that are coupled by the parallel boundary condition. Convergence is checked by increasing grid sizes until growth rates no longer change significantly, and then simulating above these thresholds.

A time window is scanned over the amplitude timetrace. At each point in the scan, growth rates are defined as $\gamma_{k}\left(t_{1}, \Delta t\right)=\ln \left[n\left(t_{1}+\Delta t\right) / n\left(t_{1}\right)\right] / \Delta t$, where $t_{1}$ is the starting point of the time window, and $\Delta t$ is the time window width. For a given $\Delta t$, the averaged growth rate $\gamma_{k}(\Delta t)$ is then defined as an average over the $\gamma_{k}\left(t_{1}, \Delta t\right)$ peaks within the time window, which smooths out any variations in the peaks which may arise due to the discrete $k_{x}$ shifts. The final $\gamma_{k}$ is then obtained by iterating this procedure and adapting $\Delta t$, until convergence is achieved when $\left|1-\gamma_{k} \Delta t\right|<\delta$. The convergence criterion has been chosen as $\delta=0.02$ for this work (which yields the same results as $\delta=0.01$ or $\delta=0.04)$. Figure $1(\mathrm{a})$, shows the mode amplitude timetrace, and the resulting calculated converged time window width $\Delta t=1 / \gamma_{k}$ between red lines. Corresponding local growth rates $\gamma_{k}(t 1, \Delta t)$ are shown in Fig. 1(b). The final growth rate in this example is the peak average $\gamma=0.33$.

For quenched modes $\gamma_{\text {avg }}<0$, there are often no clear converged or periodic growth rates, so that the $\tau_{\mathrm{AC}}$ method cannot be used. An approximate average growth rate is then obtained by using the $\gamma_{\text {avg }}$ method.

\section{APPLICATION TO SIMULATION DATA}

It was shown that the $\tau_{\mathrm{AC}}$ method works well at low values of $k_{y}$ [27]. In the present work, we further validate the approach by making apparent its advantages over alternative schemes for various plasma parameters. First, in Fig. 2 we compare the $\tau_{\mathrm{AC}}$ to the $\gamma_{\mathrm{avg}}$ method, for both adiabatic and kinetic electrons at ion and electron temperature gradients $R / L_{T}=8.75$ (in the kinetic electron case, the normalized electron temperature gradient $R / L_{T \mathrm{e}}=4$, such that we avoid a sub-dominant electron 
mode which masks the ITG quenching at high $\gamma_{E}$ ). When using adiabatic electrons, there is little difference in the linear growth rates and their reduction by flow shear as calculated by the $\tau_{\mathrm{AC}}$ and $\gamma_{\text {avg }}$ methods, with a complete quench occurring at $\alpha=\gamma_{E} / \gamma_{0} \approx 2$. However, when used in conjunction kinetic electrons, the $\tau_{\text {AC }}$ method yields promising results. The $\gamma_{\text {avg }}$ approach shows a sharp drop in growth rates at very low $\gamma_{E}$. This is a known issue, occuring even at vanishingly small $\gamma_{E}$, where physically, we would expect the impact on growth rates to also vanish. The $\tau_{\mathrm{AC}}$ method on the other hand results in a more or less linear reduction of unstable growth rates. This is much more in line with physical expectations and nonlinear simulations.

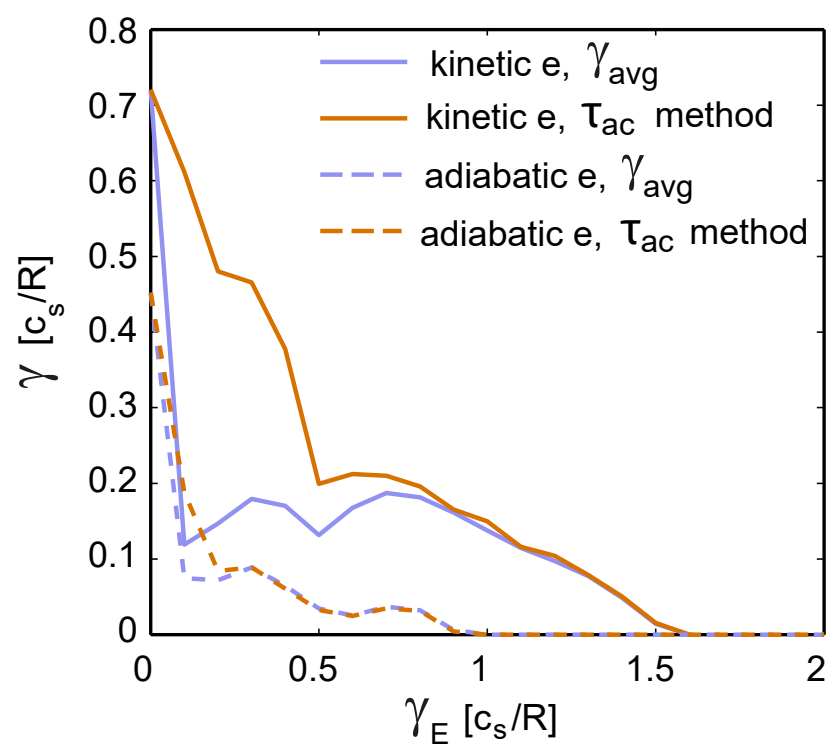

FIG. 2. Comparison of growth rates obtained with the $\gamma_{\text {avg }}$ and $\tau_{\mathrm{AC}}$ methods, with $R / L_{T \mathrm{e}}=4$ for kinetic electrons, $R / L_{T \mathrm{e}}=8.75$ for adiabatic electrons and $R / L_{T \mathrm{i}}=8.75$ for both cases.

We thus adopt the $\tau_{\mathrm{AC}}$ method and further test it by investigating the resulting quench behavior under circumstances where linear adiabatic electron approaches fail. Shown in Fig. 3 are the growth rates of linear ITG modes vs. flow shear, at both high $(\hat{s}=0.8)$ and low $(\hat{s}=0.1)$ magnetic shear. Either kinetic electrons with $R / L_{T \mathrm{i}}=6.75$ or adiabatic electrons with $R / L_{T \mathrm{i}}=10$ are used, where the temperature gradient is chosen differently to match rotationless growth rates $\gamma_{0}$ between these cases for more convenient comparison. At high $\hat{s}$, the reduction of unstable growth rates is similar between the kinetic and adiabatic electron cases, but at low $\hat{s}$, the ITG modes are quenched at much lower flow shear when adiabatic electrons are used. The adiabatic-electron case leads to a highly $\hat{s}$-dependent quench rule, with $\alpha \approx 1.4$ at $\hat{s}=0.8$ and $\alpha \approx 0.5$ at $\hat{s}=0.1$. This conflicts with the results reported in Ref. [12], where nonlinear gyrokinetic simulations using adiabatic electrons resulted in higher values around $\alpha \approx 2$, and a low dependency of $\alpha$ on magnetic shear was demonstrated. It can be concluded that linear growth rates from Floquet modes yield inaccurate results at low $\hat{s}$ when adiabatic elctrons are used, which may impact previous research done under these circumstances $[14,15]$. On the other hand, the $\tau_{\mathrm{AC}}$ method with kinetic electrons produces much better results. The quench behaviour is similar at high and low $\hat{s}$ with $\alpha \approx 1.4$ in both cases, thus demonstrating the weak dependency on $\hat{s}$.

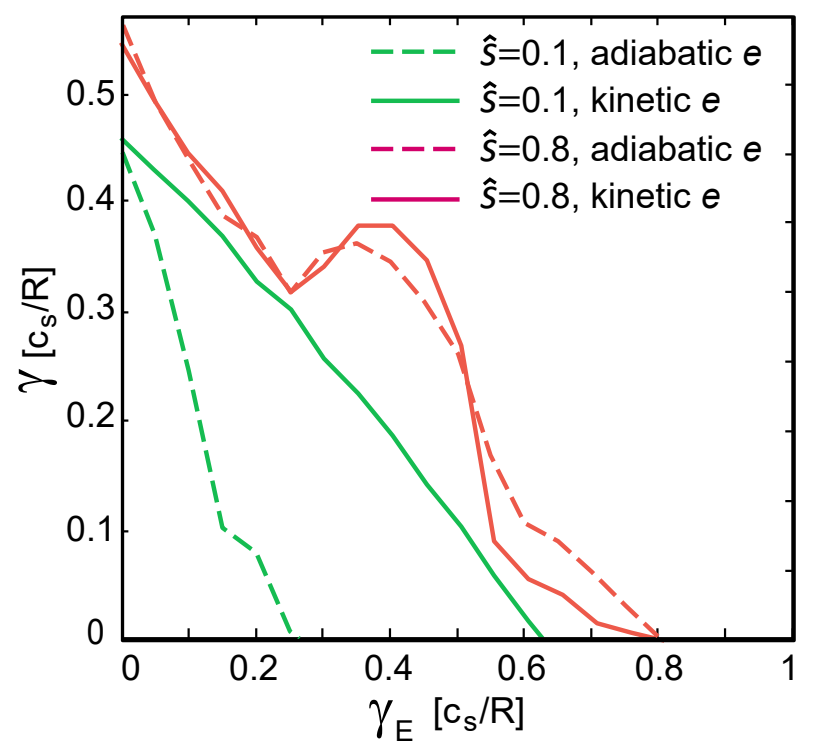

FIG. 3. Growth rates of ITG modes at two values of $\hat{s}$, for kinetic electrons (solid) with $R / L_{T \mathrm{i}}=6.75$ and adiabatic electrons (dashed) with $R / L_{T \mathrm{i}}=10$.

Next, we perform a set of nonlinear GENE simulations at the same plasma parameters as above. For the $\hat{s}=0.8$ case, simulation box sizes are $L_{x} / L_{y}=150 / 125$ with $k_{y, \min }=0.05$ and resolutions are $n_{k x} / n_{k y} / n_{z 0}=$ $128 / 16 / 16$ and $n_{v} / n_{w}=48 / 8$, while for the $\hat{s}=0.1$ case $L_{x} / L_{y}=333 / 209, k_{y, \min }=0.03, n_{k x} / n_{k y} / n_{z 0}=$ $256 / 32 / 16$ and $n_{v} / n_{w}=48 / 8$. Shown in Fig. 4 is the reduction of the ion heat flux $Q_{\mathrm{i}}$ by flow shear, again at both high and low magnetic shear. Linear rotationless growth rates in these cases are $\gamma_{0}=0.64$ and $\gamma_{0}=0.61$, respectively. When the parallel velocity gradient $(\mathrm{PVG})$ instability drive is excluded, there is clear $E \times B$ turbulence suppression for increasing $\gamma_{E}$. From these curves, we can derive an approximate quench point at $\alpha \approx 1.9$. Both the rather linear quenching behavior and the quench points are similar to the results from the $\tau_{\mathrm{AC}}$ method demonstrated in Fig. 3. As such, for weak PVG drive, by using kinetic electrons and the $\tau_{\mathrm{AC}}$ method, we overcome the issues previously encountered in obtaining physically relevant growth rates from averages over linear Floquet growth rates.

When pure toroidal rotation is included, as in the linear simulations shown in this work, no quench of fluxes is observed (for the impact of PVG in nonlinear 


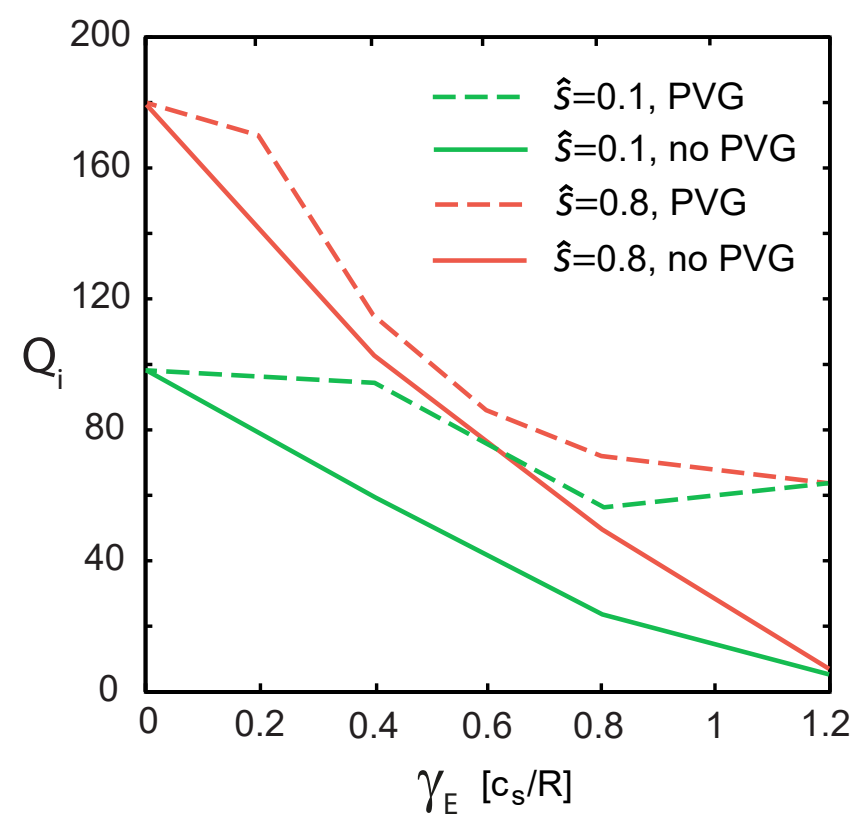

FIG. 4. Nonlinear simulations showing the reduction of the ion heat flux $\left(Q_{i}\right)$ by flow shear at high and low magnetic shear $\hat{s}$, with PVG drive either included (pure toroidal rotation) or set to zero. $R / L_{T \mathrm{e}}=4$ and $R / L_{T \mathrm{i}}=8.75$. Linear maximum growth rates are $\gamma_{0}=0.64$ for $\hat{s}=0.8$ and $\gamma_{0}=0.61$ for $\hat{s}=0.1$, leading to an approximate quench point at $\alpha=\gamma_{E} / \gamma_{0} \approx 2$.

simulations, see, e.g., Ref. [12]), different from predictions by the linear approach. Since the PVG impact is wavenumber-dependent, it can be explored if agreement is improved by by linear $E \times B$ suppression over the full spectrum and calculating quasilinear fluxes. This will be left for future work, as this concise paper focuses solely on the illustration of the $\tau_{\mathrm{AC}}$ method. Nevertheless, the significant flattening of the flux with strong PVG in nonlinear simulations is indicative of nonlinear effects which our linear approach is unable to capture. Indeed, subcritical turbulence in the high-PVG regime has already been reported $[14,15]$. In the literature $[12,13]$, despite linear stability in the presence of high $E \times B$ shear, the concomitant PVG drive leads to transient growth sufficient to sustain turbulence. Simulations initialised with sufficient-amplitude noise or fully developed turbulence were necessary to observe subcritical turbulence in such cases. This is indeed the case in our simulations, since the flow shear is only switched on following the establishment of turbulence, to save computational time during the linear phase.

Thus, this study indicates that nonlinear effects leading to a lack of justification of linear $E \times B$ stabilization models is limited to cases with significant PVG impact. This is in line with findings from quasilinear transport modeling in integrated modeling, where including the $E \times B$ stabilization in the inner half-radius (where PVG is more important) leads to an overprediction of the $E \times B$ turbulence suppression and the temperature and density profiles [28].

The $\tau_{\mathrm{AC}}$ method compares favorably to nonlinear studies on the quench rule. The original gyrofluid work $[21,22]$ indicated a value of $\alpha \approx 1$, but later studies produced higher estimates, between $\alpha=1.3$ and 2.4 (mostly between $\alpha=1.5$ and 2) [12, 24, 30,31]. The $\tau_{\mathrm{AC}}$ method in this study aligns very well with values between 1.4 and 2. As it is computationally much cheaper than nonlinear simulations, the $\tau_{\mathrm{AC}}$ method thus has significant potential to investigate the different plasma parameter dependencies of $\alpha$. This will be explored in future work.

\section{SUMMARY}

In this work, we introduced a novel growth rate calculation method for systems exhibiting Floquet oscillations, based the linear stabilization of Floquet modes, named the $\tau_{\mathrm{AC}}$ method. The approach is different from previous averages over Floquet modes, as it assumes that the averaging timescale is determined by the nonlinear decorrelation time. Furthermore, the method is used in conjunction with kinetic-electron simulations. This approach was shown to yield more physically relevant results than other linear methods, and to produce a similar quench rule of the form $\gamma_{E}=\alpha \gamma_{0}$ as found by multiple nonlinear studies in the case of weak PVG. Thus, this study indicates that in that regime, the $\tau_{\mathrm{AC}}$ method is able to capture the nonlinear quenching of ITG instability.

The $\tau_{\mathrm{AC}}$ method can be applied to calculate the plasma parameter dependencies of the $\alpha$ factor, potentially leading to a simple but accurate method for determining the impact of flow shear on ITG instability. Such a computationally inexpensive method may be deployed in quasilinear transport models such as QuaLiKiz [28, 29], which will be explored in future work.

\section{ACKNOWLEDGEMENTS}

This work has been carried out within the framework of the EUROfusion Consortium and has received funding from the Euratom research and training program 20142018 under grant agreement No. 633053. The views and opinions expressed herein do not necessarily reflect those of the European Commission.
[1] E. J. Doyle, W. A. Houlberg, Y. Kamada, V. Mukhovatov, T. H. Osborne, A. Polevoi, G. Bateman, J. W. Con- nor, J. G. Cordey, T. Fujita, et al., Nucl. Fusion 47, S18 
(2007)

[2] F. Romanelli, Phys. Fluids B 1, 1018 (1989)

[3] S. C. Guo and F. Romanelli, Phys. Fluids B 5, 520 (1993)

[4] C. Bourdelle, J. Citrin, B. Baiocchi, A. Casati, P. Cottier, X. Garbet, F. Imbeaux and JET Contributors, Plasma Phys. Control. Fusion 58, 014036 (2016)

[5] T. Dannert and F. Jenko, Phys. Plasmas 12, 072309 (2005)

[6] F. Jenko, T. Dannert, and C. Angioni, Plasma Phys. Control. Fusion 47, B195 (2005)

[7] F. Merz and F. Jenko, Nucl. Fusion 50, 054005 (2010)

[8] K. H. Burrell, Phys. Plasmas 4, 1499 (1997)

[9] P. A. Politzer, C. C. Petty, R.J. Jayakumar, T. C. Luce, M. R. Wade, J. C. DeBoo, J. R. Ferron, P. Gohil, C. T. Holcomb, A. W. Hyatt, et al., Nucl. Fusion 48, 075001 (2008)

[10] H. Biglari and P. H. Diamond, Phys. Plasmas 2, 1 (1990)

[11] S. L. Newton, S.C. Cowley, and N.F. Loureiro, Plasma Phys. Control. Fusion 52, 125001 (2010)

[12] J. E. Kinsey, R. E. Waltz, and J. Candy, Phys. Plasmas 12, $062302(2005)$

[13] M. Barnes, F. I. Parra, and A. A. Schekochihin, Phys. Rev. Lett. 106, 175004 (2011)

[14] E. G. Highcock, M. Barnes, A. A. Schekochihin, F. I. Parra, C. M. Roach, and S. C. Cowley, Phys. Rev. Lett. 105, 215003 (2010)

[15] E. G. Highcock, A. A. Schekochihin, S. C. Cowley, M. Barnes, F. I. Parra, C. M. Roach, and W. Dorland, Phys. Rev. Let. 109, 265001 (2012)

[16] F. Jenko, W. Dorland, M. Kotschenreuter and B. N. Rogers, Phys. Plasmas 7, 1904 (2000); for code details and access, see http://www.genecode.org

[17] F. Merz, Gyrokinetic Simulation of Multimode Plasma Turbulence, Ph.D. thesis, University of Münster (2009)

[18] A. J. Brizard, Rev. Mod. Phys. 79, 421 (2007)

[19] D. Told, Gyrokinetic Microturbulence in Transport Barriers, Ph.D. thesis, University of Ulm (2012)
[20] G. W. Hammett, W. Dorland, N. F. Loureiro, and T. Tatsuno. Proceedings of the 48th Annual Meeting of the APS Division of Plasma Physics, (American Physical Society, Philadelphia, 2006).

[21] R. E. Waltz, G. D. Kerbel, and J. Milovich, Phys. Plasmas 1, 2229 (1994)

[22] R. E. Waltz, G. D. Kerbel, J. Milovich, and G. W. Hammett, Phys. Plasmas 2, 2408 (1995)

[23] G. M. Staebler, R. E. Waltz, J. Candy, and J. E. Kinsey, Phys. Rev. Lett. 110, 055003 (2013)

[24] R. E. Waltz, R. L. Dewar, and X. Garbet, Phys. Plasmas 5, $1784(1998)$

[25] C. M. Roach, I. G. Abel, R. J. Akers, W. Arter, M. Barnes, Y. Camenen, F. J. Carsson, G. Colyer, J. W. Connor, A. C. Cowley, et al., Plasma Phys. Control. Fusion 51, 124020 (2009)

[26] P. Cottier, C. Bourdelle, Y. Camenen, Ö. D. Gürcan, F. J. Casson, X. Garbet, P. Hennequin, and T. Tala, Plasma Phys. Control. Fusion 56, 015011 (2014)

[27] J. Citrin, C. Bourdelle, P. Cottier, D. F. Escande, Ö. D. Gürcan, D. R. Hatch, G. M. D. Hogeweij, F. Jenko, and M. J. Pueschel, Phys. Plasmas 19, 062305 (2012)

[28] J. Citrin, C. Bourdelle, F. J. Casson, C. Angioni, N. Bonanomi, Y. Camenen, X. Garbet, L. Garzotti, T. Görler, Ö. D. Gürcan et al., Plasma Phys. Control. Fusion 59, 124005 (2017)

[29] C. Bourdelle, X. Garbet, F. Imbeaux, A. Casati, N. Dubuit, R. Guiret, and T. Parisot, Phys. Plasmas 14, 112501 (2007)

[30] R. E. Waltz, J. M. Candy, and M. N. Rosenbluth, Phys. Plasmas 9,1938 (2002)

[31] A. M. Dimits, B. I. Cohen, W. M. Nevins, and D. E. Shumaker, Proceedings of the Eighteenth IAEA Fusion Energy Conference, (International Atomic Energy Agency, Sorrento, 2000). 
(a)

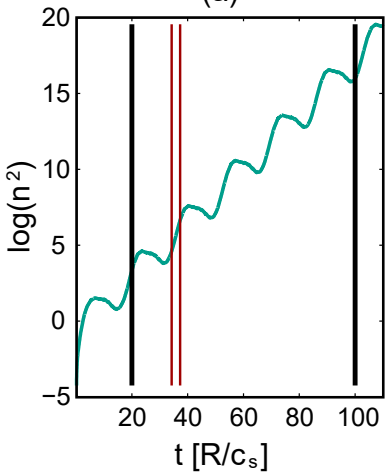

(b)

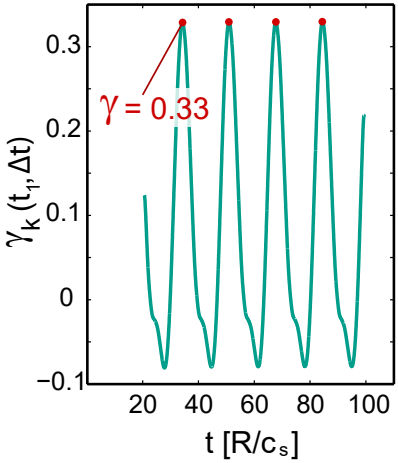




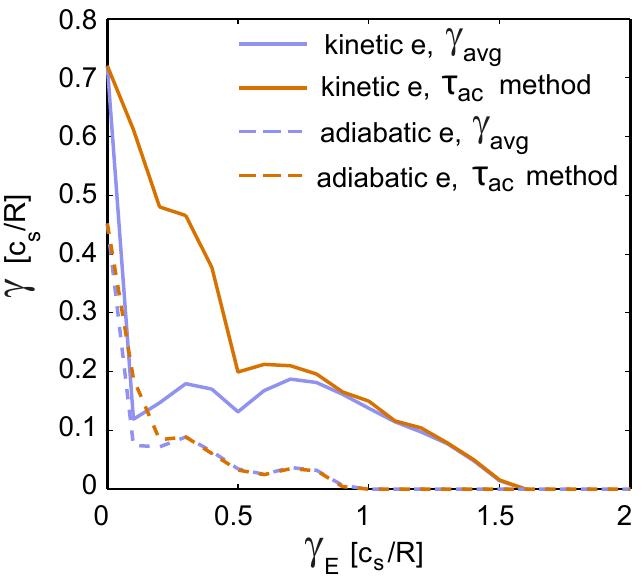




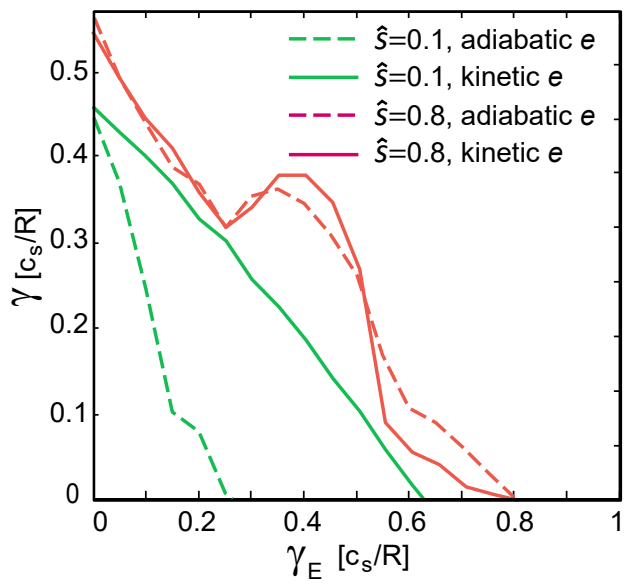




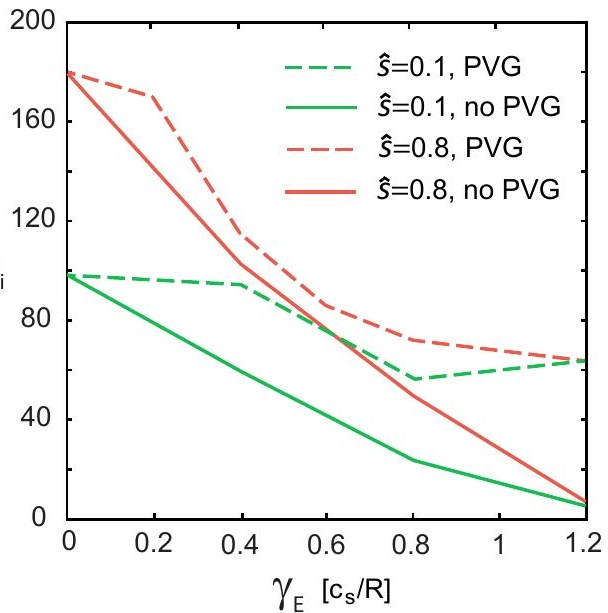

